\title{
Hormônio antimülleriano como marcador de fertilidade em fêmeas suínas
} Anti-Müllerian hormone as a fertility marker in swine females

\author{
Carolini Schultz ${ }^{1}$, Pricila Baldessar ${ }^{2}$, Camila Ribeiro Carvalho de Brito ${ }^{3}$, Ivan Bianchi ${ }^{1,2}$ \\ ${ }^{1}$ Instituto Federal Catarinense Campus Araquari, Núcleo de Pesquisa, Ensino e Extensão em Produção Animal \\ (NEPPA), BR $280 \mathrm{Km}$ 27, 89245-000, Araquari, SC, Brasil \\ ${ }^{2}$ Instituto Federal Catarinense Campus Araquari, Mestrado Profissional em Produção e Sanidade Animal (PGPSA), \\ BR $280 \mathrm{Km}$ 27, 89245-000, Araquari, SC, Brasil \\ ${ }^{3}$ Universidade Federal de Pelotas Campus Capão do Leão, Núcleo de Ensino e Pesquisa em Reprodução Animal \\ (ReproPEL), Faculdade de Veterinária, Campus Universitário S/Nº, 96010-900, Capão do Leão, RS, Brasil
}

\section{Resumo}

O hormônio antimülleriano é conhecido por seu papel na regressão dos ductos de Müller durante a fase de diferenciação sexual dos machos. Ainda assim, esse hormônio atua de forma ainda mais ampla no desenvolvimento de ambos os sexos. O hormônio antimülleriano é diferencialmente expresso em machos e fêmeas durante toda a vida. Nas fêmeas esse hormônio é produzido pelas células da granulosa dos folículos ovarianos e atua modulando os processos de secreção de gonadotrofinas, foliculogênese e esteroidogênese, sendo considerado um biomarcador confiável para fertilidade em diferentes espécies. Apesar de pouquíssimo estudado na espécie suína, alguns trabalhos vêm demonstrando a existência de associações entre os níveis séricos do hormônio antimülleriano e os fenótipos de maior fertilidade. De forma que, o objetivo desta revisão é fazer um levantamento do conhecimento já acumulado acerca da temática, demostrando a importância do desenvolvimento de novos estudos; tendo em vista que o aprofundamento do conhecimento sobre as funções do hormônio antimülleriano nas fêmeas da espécie suína pode contribuir para o melhor entendimento da fisiologia da espécie

Palavras-chave: AMH, biomarcador molecular, poliovulatório, puberdade, precocidade sexual

\section{Abstract}

The anti-Müllerian hormone (AMH) is known for its role in the regression of Muller ducts during the phase of sexual differentiation in males. Notwithstanding, this hormone acts more broadly on the development of both sexes. The AMH is differentially expressed both in males and females throughout life. In females, this hormone is produced by granulosa cells of ovarian follicles and modulates the gonadotropin secretion, folliculogenesis, and steroidogenesis, and is considered a reliable fertility biomarker in different species. Despite the lack of studies in the swine species, some reports demonstrated the association between serum levels of AMH and more fertile phenotypes. Therefore, this review aims to survey the current knowledge about this theme, demonstrating the importance of the development of new research, since the strengthening of the knowledge on the functions of the AMH in swine females may contribute to understanding the physiology of the species.

Keywords: AMH, molecular biomarker, polyovulatory, puberty, sexual precocity.

\section{Introdução}

O hormônio antimülleriano $(\mathrm{AMH})$ é um dímero glicoproteico pertencente ao grupo dos fatores de transformação e crescimento beta (TGF- $\beta$ ). O próprio nome já indica, que esse hormônio é amplamente conhecido por sua atuação, durante a fase de diferenciação sexual dos machos, na regressão dos ductos paramesonéfricos ou ductos de Müller (Cate et al., 1986), isto é, ele atua na regressão das estruturas que nas fêmeas, darão origem aos ovidutos, cornos uterinos, corpo do útero, cervix e vagina. Contudo, mais recentemente, surgiram evidências de que o AMH contribui de forma ainda mais ampla ao desenvolvimento dos mamíferos, modulando em ambos os sexos, de maneira sexualmente dimórfica, a formação do trato reprodutivo e de partes do sistema nervoso central (Wang et al., 2009; Wittmann e McLennan, 2013; Malone et al., 2019).

O AMH é produzido com maior intensidade pelas células de Sertoli e pelas células da camada da granulosa dos folículos ovarianos em machos e fêmeas, respectivamente (Cate et al., 1986; Josso e 
Clemente, 2003). Mas também pode ser expresso em pequenas quantidades por neurônios (Cimino et al., 2016; Kereilwe e Kadokawa, 2020), células secretoras da hipófise (Garrel et al., 2016; Kereilwe et al., 2018; Garrel et al., 2019) e outros tipos celulares; sendo que, quando produzido fora das gônadas, atua como um fator de crescimento no controle autócrino e parácrino (Garrel et al., 2016; Malone et al., 2019).

Por meio de receptores específicos, os receptores para o hormônio antimülleriano do tipo 2 (AMHRII), o AMH atua regulando durante o período embrionário a migração dos neurônios secretores de GnRH, a formação do hipotálamo e do bulbo olfatório, a ramificação de neurônios associados ao órgão vomeronasal e ao epitélio olfatório e no desenvolvimento do trato reprodutivo (Malone et al., 2019). Além disso, pelo fato de o AMH e AMHRII serem diferencialmente expressos em machos e fêmeas, acredita-se que o AMH junto a testosterona seja responsável pela masculinização do centro pré-ovulatório do hipotálamo e outros centros sexualmente dimórficos do sistema nervoso central (Wang et al., 2009; Wittmann e McLennan, 2013).

Após o nascimento, e durante toda a vida, o AMH atua modulando os processos de gametogênese, esteroidogênese e também a produção de hormônio liberador de gonadotrofinas $(\mathrm{GnRH})$, hormônio folículo estimulante (FSH) e hormônio luteinizante (LH) (Cimino et al., 2016; Garrel et al., 2016; Kereilwe et al., 2018; Garrel et al., 2019). Além disso, a ocorrência de mutações que acarretam perda de função tanto do AMH quando dos AMHRII, resultam em fenótipos com fertilidade reduzida e infertilidade causados por deficiência na secreção de gonadotrofinas, distúrbio denominado de hipogonadismo hipogonadotrófico (Malone et al., 2019).

Pelo fato de o AMH ser um biomarcador confiável para a fertilidade em mulheres, com aplicações práticas na clínica, reprodução assistida e no diagnóstico de diferentes distúrbios ovarianos como a exaustão precoce da reserva de folículos primordiais (Moolhuijsen e Visser, 2020), tem-se despertado o interesse em averiguar se esse hormônio também pode ter aplicações como biomarcador na reprodução animal, tanto na área de reprodução de espécies pecuárias (Ireland et al., 2011; Monniaux et al., 2012; Steel et al., 2018; Umer et al., 2019) quanto na área de conservação de espécies em risco de extinção (Wilson et al., 2011; Pollock et al., 2020).

Nos ruminantes domésticos, especialmente em fêmeas bovinas, o AMH é um biomarcador positivamente correlacionado com a contagem de folículos antrais (AFC), com a reserva ovariana (Ireland et al., 2011; Monniaux et al., 2012) e com a capacidade de responder a protocolos de superovulação (Mossa et al., 2017; Umer et al., 2019); e negativamente correlacionado com a idade a puberdade (ElSheikh Ali et al., 2017). Nessas espécies, uma única mensuração sérica do hormônio é suficiente para avaliar o potencial reprodutivo da fêmea, já que apesar da alta variabilidade entre indivíduos, em um mesmo animal os níveis plasmáticos do hormônio se mantêm constantes e são altamente duplicáveis (Ireland et al., 2011; Monniaux et al., 2012). Em fêmeas da espécie suína, contudo, são pouquíssimos os estudos acerca da temática. Objetivou-se com esse trabalho realizar um levantamento acerca do conhecimento acumulado até o presente momento.

\section{Particularidades do AMH nas fêmeas suínas}

Sabe-se que nas fêmeas da espécie suína o AMH começa a ser produzido nas células da granulosa já no final do primeiro mês de vida, quando se inicia a fase de foliculogênese (Monniaux et al., 2014). Contudo, diferentemente do que ocorre nas demais espécies de interesse pecuário, nas fêmeas suínas o AMH não é expresso somente pelas células da teca, sendo produzido também, em menor quantidade, pelas células da teca e pelas células dos corpos lúteos (Almeida et al., 2018). De forma que esse hormônio é produzido por folículos de todas as etapas de desenvolvimento, excetuando-se os folículos primordiais e folículos atrésicos (Almeida et al., 2018).

$\mathrm{O}$ AMH é produzido em maior quantidade nos pequenos folículos recém-recrutados e, em menor quantidade, também pelos folículos pré-ovulatórios e pelos corpos lúteos, regulando diferentes etapas da foliculogênese (Almeida et al., 2018; Li et al., 2020). Nas células da granulosa, o AMH inibe a transcrição dos receptores de FSH e, consequentemente, inibe a produção de aromatase e estradiol dependentes de FSH. Nas células da teca, o AMH inibe a transcrição dos receptores de LH e, consequentemente, a produção de diferentes enzimas responsáveis pela produção de hormônios esteroides (Li et al., 2020).

Dessa maneira, o AMH possui função essencialmente inibitória. Por meio de atuação parácrina e autócrina ainda pouco esclarecidas, o AMH inibe o recrutamento dos folículos primordiais e, por intermédio de um mecanismo de down-regulation sobre a produção de receptores de FSH e receptores de LH, também inibe a diferenciação e o crescimento dos folículos recrutados (Almeida et al., 2018; Li et 
al., 2020). Ainda assim, quando se compara a produção ovariana de AMH nas fêmeas suínas com à de fêmeas de outras espécies pecuárias, observa-se que a produção ovaria de $\mathrm{AMH}$ nas fêmeas suínas é muito menor (Monniaux et al., 2012; Estienne et al., 2020), o que indica que os folículos das fêmeas suínas são menos inibidos pelo AMH (Estienne et al., 2020). As concentrações intra-foliculares do AMH na espécie suína são 1000 vezes menores do que as observadas nas fêmeas bovinas. Isso ocorre devido a menor atividade da região gênica que promove a transcrição do gene que codifica o AMH, provavelmente em razão do acúmulo de deleções, e devido à expressão de SMAD6, fator que inibe a rota de produção do AMH nas células da granulosa (Estienne et al., 2020). Dessa maneira, justifica-se o fenótipo poliovulatório da espécie suína, já que um número maior de folículos primordiais é ativado, esses folículos são mais responsivos ao estímulo do FSH e menos sensíveis às suas flutuações e, consequentemente, um número maior de folículos é selecionado e se tornam aptos a ovular (Estienne et al., 2020; Li et al., 2020).

Contudo, quando se compara fêmeas suínas com diferentes concentrações séricas do AMH, observa-se que, ao passo que as fêmeas com menores concentrações séricas do hormônio apresentam maior reserva ovariana (Steel et al., 2018; Tanihara et al., 2019), também apresentam menor número de folículos antrais quando comparado às fêmeas com maiores concentrações séricas do hormônio (Tanihara et al., 2019). Isso demonstra que, em conformidade com o que foi descrito em outras espécies, o AMH atua inibindo a atresia folicular nas fêmeas suínas (Almeida et al., 2018).

A produção do $\mathrm{AMH}$ pelos corpos lúteos aparenta ser uma característica única da espécie suína e, de acordo com Almeida et al. (2018), esse hormônio atuaria inibindo a sensibilidade ao FSH e, consequentemente, inibindo o recrutamento precoce dos pequenos folículos antrais. Isso porque na espécie suína, logo após a ovulação, cessa-se o feedback negativo do estradiol sobre o FSH e os corpos lúteos, produtores de progesterona, só adquirem capacidade funcional adequada após o $8^{\circ}$ dia do ciclo, de maneira que o feedback-negativo da progesterona sobre o hormônio liberador de gonadotrofinas $(\mathrm{GnRH})$ demora a se estabelecer (Soede et al., 2011).

\section{AMH como biomarcador para precocidade sexual em leitoas}

Logo, para a espécie suína, ao passo que as concentrações séricas do AMH estão diretamente relacionadas à AFC (Tanihara et al., 2019), a mesma relação não pode ser estabelecida quando se trata da reserva de folículos antrais (Steel et al., 2018; Tanihara et al., 2019). Essa discordância se deve ao fato de que, ao contrário do que ocorre em fêmeas bovinas, não foram estabelecidas correlações consistentes entre a capacidade ovariana e a AFC (Warren et al., 2015). Ainda assim, alguns estudos vêm demonstrando associações entre as concentrações séricas do hormônio e fenótipos de maior precocidade sexual (Van Wettere et al., 2015; Am-In et al., 2020).

Ao avaliar leitoas com diferentes fenótipos para idade a puberdade, Am-In et al. (2020), observaram que as concentrações séricas para o $\mathrm{AMH}$ aos 90, 120 e 150 dias de idade foram negativamente correlacionadas à idade a puberdade e, quando se compara leitoas que expressam o estro puberal espontaneamente antes dos 200 dias de idade com leitoas que permanecem em anestro nesse período; durante o período pré-púbere as leitoas que ciclam apresentam níveis séricos para o $\mathrm{AMH}$ significativamente superiores. Além disso, leitoas com maiores concentrações séricas de AMH respondem mais precocemente aos protocolos de indução do estro puberal com o uso de gonadotrofinas exógenas (PG600®) (Am-In et al., 2020) e também tendem a apresentar melhor resposta ao estímulo do cachaço durante a fase de estimulação (Van Wettere et al., 2015). Tais achados evidenciam o papel do AMH como um biomarcador preditivo para a precocidade sexual em fêmeas suínas.

Esses estudos acerca da utilização do AMH como um possível biomarcador para precocidade sexual em fêmeas suínas encontram precedente em estudos com roedores, em que se demonstrou que o AMH apresenta importante papel na regulação central da reprodução, com implicações também no processo de aquisição da puberdade em fêmeas, visto que os AMHRII são diferencialmente expressos nos corpos celulares dos neurônios GnRH em fêmeas pré-púberes e fêmeas cíclicas (Garrel et al., 2016). O AMH produzido pelos ovários regula, de maneira dose dependente, a pulsatilidade dos neurônios GnRH (Cimino et al., 2016) e o pulso de secreção do GnRH modula a expressão de AMHRII presente nos gonadotrofos, células secretoras de gonadotrofinas localizadas na hipófise (Garrel et al., 2019).

Em ratas pré-púberes, a administração exógena de AMH promove a expressão e secreção de FSH pelos gonadotrofos (Garrel et al., 2016). Já em ratas cíclicas, com alta frequência pulsátil de GnRH, a administração exógena de AMH promove o aumento da secreção de LH (Cimino et al., 2016; Garrel et al., 2019). Em leitoas cíclicas, o AMH atua inibindo a expressão de receptores de FSH pelas células da 
granulosa e, consequentemente, inibe também a produção de aromatase e estradiol dependentes de FSH (Li et al., 2020). Contudo, em leitoas pré-púberes o AMH aparenta não interferir na produção de estradiol, já que nas semanas que antecedem a expressão do primeiro estro ocorre aumento conjunto na produção de AMH e estradiol pelas células da granulosa (Am-In et al., 2020).

Leitoas púberes com altas concentrações de $\mathrm{AMH}$ apresentam maior AFC, maior número de oócitos coletados e maior qualidade oocitária (Tanihara et al., 2019). Apesar da grande diferença genética entre as linhagens avaliadas por Tanihara et al. (2019), o melhor desempenho das fêmeas Large White poderia ser explicado, em parte, pelo fato de que o AMH estimula os neurônios GnRH de maneira dose dependente e, consequentemente, quanto maior as concentrações séricas do AMH maior é a secreção de LH (Cimino et al., 2016). Fêmeas com maiores níveis séricos de LH apresentam maior capacidade ovulatória (Cimino et al., 2016), já que o LH é responsável pelo crescimento final, maturação e ovulação dos folículos (Geisert et al., 2020).

Por fim, o AMH é um assunto pouco estudado na espécie suína, mas os trabalhos publicados reforçam a hipótese de que esse hormônio pode ser um biomarcador preditivo para a fertilidade nas fêmeas suínas. $\mathrm{O} \mathrm{AMH}$ atua inibindo a atresia dos folículos ovarianos e possui relação direta com o número de folículos antrais e com a capacidade ovulatória da fêmea. Além disso, fêmeas com fenótipos de maior precocidade sexual apresentam maiores níveis séricos de AMH entre os 90 e 150 dias de idade. De forma que a dosagem do hormônio nesse período facilitaria a seleção de leitoas com maior potencial produtivo. Todavia, os mecanismos pelos quais o AMH estimula a expressão e secreção de gonadotrofinas a nível central e inibe a ação desses hormônios a nível periférico precisam ser melhor esclarecidos. De forma que estudos mais robustos devem ser realizados a fim de suplementar o conhecimento até agora acumulado e validar uma metodologia para a mensuração esse hormônio em diferentes grupos de fêmeas para que sejam estabelecidos valores de referência na espécie suína.

\section{Considerações finais}

Os conhecimentos acerca das funções do AMH na espécie suína ainda são incipientes, contudo, o aprofundamento desse conhecimento pode ser de importância para o entendimento da fisiologia básica da espécie e contribuir para a seleção das leitoas de reposição, visto que já foram estabelecidas associações entre a ocorrência do primeiro estro e as concentrações séricas do hormônio em leitoas em crescimento.

\section{Agradecimentos}

À Fundação de Amparo à Pesquisa e Inovação do Estado de Santa Catarina - FAPESC, agradecemos pelo suporte.

\section{Referências}

Almeida F, Costermans NGJ, Soede NM, Bunschoten A, Keijer J, Kemp B, Teerds KJ. Presence of anti-Mullerian hormone (AMH) during follicular development in the porcine ovary. PLoS One, v.13, n.7, p.e0197894, 2018.

Am-In N, Suwimonteerabutr J, Kirkwood RN. Serum Anti-Mullerian Hormone and Estradiol Concentrations in Gilts and Their Age at Puberty. Animals (Basel), v.10, n.11, 2020.

Cate RL, Mattaliano RJ, Hession C, Tizard R, Farber NM, Cheung A, Ninfa EG, Frey AZ, Gash DJ, Chow EP, et al. Isolation of the bovine and human genes for Mullerian inhibiting substance and expression of the human gene in animal cells. Cell, v.45, n.5, p.685-698, 1986.

Cimino I, Casoni F, Liu X, Messina A, Parkash J, Jamin SP, Catteau-Jonard S, Collier F, Baroncini M, Dewailly D, Pigny P, Prescott M, Campbell R, Herbison AE, Prevot V, Giacobini P. Novel role for anti-Mullerian hormone in the regulation of $\mathrm{GnRH}$ neuron excitability and hormone secretion. Nat Commun, v.7, p.10055, 2016.

El-Sheikh Ali H, Kitahara G, Takahashi T, Mido S, Sadawy M, Kobayashi I, Hemmi K, Osawa T. Plasma anti-Mullerian hormone profile in heifers from birth through puberty and relationship with puberty onset. Biol Reprod, v.97, n.1, p.153-161, 2017.

Estienne A, Jarrier P, Staub C, Venturi E, Le Vern Y, Clemente N, Monniaux D, Monget P. AntiMullerian hormone production in the ovary: a comparative study in bovine and porcine granulosa 
cellsdagger. Biol Reprod, v.103, n.3, p.572-582, 2020.

Garrel G, Denoyelle C, L'Hote D, Picard JY, Teixeira J, Kaiser UB, Laverriere JN, CohenTannoudji J. GnRH Transactivates Human AMH Receptor Gene via Egr1 and FOXO1 in Gonadotrope Cells. Neuroendocrinology, v.108, n.2, p.65-83, 2019.

Garrel G, Racine C, L'Hote D, Denoyelle C, Guigon CJ, di Clemente N, Cohen-Tannoudji J. AntiMullerian hormone: a new actor of sexual dimorphism in pituitary gonadotrope activity before puberty. Sci Rep, v.6, p.23790, 2016.

Geisert RD, Sutvosky P, Lucy MC, Bartol FF, Meyer AE. Reproductive physiology of swine, In: Bazer FW, Lamb GC, Wu G (Eds.) Animal Agriculture: Sustainability, Challenges and Innovations. Academic Press, p.263-281, 2020.

Ireland JJ, Smith GW, Scheetz D, Jimenez-Krassel F, Folger JK, Ireland JL, Mossa F, Lonergan P, Evans AC. Does size matter in females? An overview of the impact of the high variation in the ovarian reserve on ovarian function and fertility, utility of anti-Mullerian hormone as a diagnostic marker for fertility and causes of variation in the ovarian reserve in cattle. Reprod Fertil Dev, v.23, n.1, p.1-14, 2011. Josso N, Clemente N. Transduction pathway of anti-Mullerian hormone, a sex-specific member of the TGF-beta family. Trends Endocrinol Metab, v.14, n.2, p.91-97, 2003.

Kereilwe O, Kadokawa H. Anti-Mullerian hormone and its receptor are detected in most gonadotropinreleasing-hormone cell bodies and fibers in heifer brains. Domest Anim Endocrinol, v.72, p.106432, 2020.

Kereilwe O, Pandey K, Borromeo V, Kadokawa H. Anti-Mullerian hormone receptor type 2 is expressed in gonadotrophs of postpubertal heifers to control gonadotrophin secretion. Reprod Fertil Dev, v.30, n.9, p.1192-1203, 2018.

Li Y, Gao D, Xu T, Adur MK, Zhang L, Luo L, Zhu T, Tong X, Zhang D, Wang Y, Ning W, Qi X, Cao Z, Zhang Y. Anti-Mullerian hormone inhibits luteinizing hormone-induced androstenedione synthesis in porcine theca cells. Therio, v.142, p.421-432, 2020.

Malone SA, Papadakis GE, Messina A, Mimouni NEH, Trova S, Imbernon M, Allet C, Cimino I, Acierno J, Cassatella D, Xu C, Quinton R, Szinnai G, Pigny P, Alonso-Cotchico L, Masgrau L, Marechal JD, Prevot V, Pitteloud N, Giacobini P. Defective AMH signaling disrupts GnRH neuron development and function and contributes to hypogonadotropic hypogonadism. Elife, v.8, p.e47198, 2019.

Monniaux D, Clement F, Dalbies-Tran R, Estienne A, Fabre S, Mansanet C, Monget P. The ovarian reserve of primordial follicles and the dynamic reserve of antral growing follicles: what is the link? Biol Reprod, v.90, n.4, p.85, 2014.

Monniaux D, Drouilhet L, Rico C, Estienne A, Jarrier P, Touze JL, Sapa J, Phocas F, Dupont J, Dalbies-Tran R, Fabre S. Regulation of anti-Mullerian hormone production in domestic animals. Reprod Fertil Dev, v.25, n.1, p.1-16, 2012.

Moolhuijsen LME, Visser JA. Anti-Mullerian Hormone and Ovarian Reserve: Update on Assessing Ovarian Function. J Clin Endocrinol Metab, v.105, n.11, p.3361-3373, 2020.

Mossa F, Jimenez-Krassel F, Scheetz D, Weber-Nielsen M, Evans ACO, Ireland JJ. Anti-Mullerian Hormone (AMH) and fertility management in agricultural species. Reproduction, v.154, n.1, p.R1-R11, 2017.

Pollock KE, O'Brien JK, Roth TL, Proudfoot J, Niederlander J, Micheas L, Robeck TR, Stoops MA. Anti-Mullerian hormone in managed African and Asian rhino species. Gen Comp Endocrinol, v.294, p.113487, 2020.

Soede NM, Langendijk P, Kemp B. Reproductive cycles in pigs. Anim Reprod Sci, v.124, n.3-4, p.251258, 2011.

Steel A, Athorn RZ, Grupen CG. Anti-Mullerian hormone and Oestradiol as markers of future reproductive success in juvenile gilts. Anim Reprod Sci, v.195, p.197-206, 2018.

Tanihara F, Hirata M, Iizuka S, Sairiki S, Nii M, Nguyen NT, Le QA, Hirano T, Otoi T. Relationship among ovarian follicular status, developmental competence of oocytes, and anti-Mullerian hormone levels: A comparative study in Japanese wild boar crossbred gilts and Large White gilts. Anim Sci J, v.90, n.6, p.712-718, 2019.

Umer S, Zhao SJ, Sammad A, Weldegebriall Sahlu B, Yunwei P, Zhu H. AMH: Could It Be Used as A Biomarker for Fertility and Superovulation in Domestic Animals? Genes (Basel), v.10, n.12, 2019.

Van Wettere W, Weaver A, Staveley L, Muller T, Hewitt R, Hughes P, Kirkwood R. Prenatal and neonatal gilt management and anti-Müllerian hormone: effects on the ovary and response to the boar. Anim Prod Sci, v.55, n.12, p.1515-1515, 2015. 
Wang PY, Protheroe A, Clarkson AN, Imhoff F, Koishi K, McLennan IS. Mullerian inhibiting substance contributes to sex-linked biases in the brain and behavior. Proc Natl Acad Sci U S A, v.106, n.17, p.7203-7208, 2009.

Warren L, Murawski M, Wilk K, Zieba DA, Bartlewski PM. Suitability of antral follicle counts and computer-assisted analysis of ultrasonographic and magnetic resonance images for estimating follicular reserve in porcine, ovine and bovine ovaries ex situ. Exp Biol Med (Maywood), v.240, n.5, p.576-584, 2015.

Wilson RC, Reynolds III JE, Wetzel DL, Schwierzke-Wade L, Bonde RK, Breuel KF, Roudebush WE. Secretion of anti-Müllerian hormone in the Florida manatee Trichechus manatus latirostris, with implications for assessing conservation status. Endangered Species Research, v.14, n.2, p.107-112, 2011.

Wittmann W, McLennan IS. Anti-Mullerian hormone may regulate the number of calbindin-positive neurons in the sexually dimorphic nucleus of the preoptic area of male mice. Biol Sex Differ, v.4, n.1, p.18, 2013. 